Die aggressiven Thymome (WHOTyp $\mathrm{B}_{2}$ und $\mathrm{B}_{3}$ ) und Thymuskarzinome einschließlich der neuroendokrinen Karzinome (z. B. Thymuskarzinoide) ähneln in der radiologischen Darstellung den malignen Keimzelltumoren und den Lymphomen. Die oft nicht gekapselten Tumoren wachsen ausladend, und die Karzinome zeigen ganz im Gegensatz zu den Thymomen häufig (ca. in 30\% der Fälle) einen Lymphknotenbefall supraklavikulär oder im mittleren Mediastinum. Die Bestimmung der Tumormarker für Keimzelltumoren ist in diesen Fällen bei nichtseminomatösen Tumoren richtungsweisend. In der Regel werden die Lymphome systemisch, die übrigen Tumortypen je nach Resektabilität multimodal behandelt. Die exakte histologische Zuordnung ist deshalb vor der Therapie unabdingbar.

\section{Mittleres Mediastinum}

In diesem Kompartiment überwiegen die Lymphknotenmetastasen von Lungenkarzinomen. Lässt man diese Gruppe sowie andere Lungenerkrankungen mit Lymphknotenbeteiligung wie die Tuberkulose außer Betracht, so überwiegen die Sarkoidose, Lymphknotenmetastasen extrathorakaler Karzinome (Mamma-, kolorektales und Nierenzellkarzinom) sowie primäre Lymphome. Durch transtracheale bzw. transösophageale ultraschallgestützte Biopsie oder Videomediastinoskopie wird die histologische Differenzialdiagnose möglich. Bronchogene Zysten und Perikardzysten lassen sich im MRT gut differenzieren. Tumoren in Beziehung zum Ösophagus lassen an Leiomyome, GIST-Tumoren, Granularzelltumoren und Ösophaguskarzinome denken und werden im Rahmen der Ösophagoskopie mit endoskopischem Ultraschall abgeklärt. In diesem Kompartiment treten auch die meisten gutartigen Tumoren des Bindegewebes (vor allem Lipome, $\bullet$ Abb. 6) auf. Im Mediastinum kommen so gut wie alle Sarkomtypen vor, sind aber insgesamt sehr selten (am häufigsten das Liposarkom) und stellen aufgrund der anatomischen Umgebung und der Bedeutung der radikalen Resektion mit entsprechendem Sicher-

\title{
Nierenarterienstenose und Phäochromozytom
}

\author{
Eine Patientin mit einer Nierenarterienstenose kam zwei Jahre nach einer Hyper- \\ tonieabklärung zur Durchführung einer Angiographie in das Klinikum. Aus den \\ Untersuchungen leitete sich der Verdacht auf ein Phäochromozytom ab.
}

Eine 46-jährige Patientin wurde zur Abklärung einer Hypertonie vorgestellt. Sonographisch ergab sich der Befund einer parenchymverschmälerten linken Niere. Szintigraphisch wies diese einen 20\%igen Funktionsanteil auf. Zur weiteren Abklärung erfolgte eine MR-Angiographie, woraus sich der Verdacht auf eine höhergradige Nierenarterienstenose ableitete. Dieser Verdacht wurde duplexsonographisch erhärtet. Die empfohlene i.a.-Angiographie wurde dann aufgrund der Diagnose eines follikulären Schilddrüsenkarzinoms zurückgestellt. Nach Thyreoidektomie, zweimaliger Radioiodtherapie und letztlich unauffälliger Nachsorge erfolgte zwei Jahre später eine erneute duplexsonographische Kontrolle der Nierenarterien. Bei dieser ergab sich kein eindeutiger Stenosenachweis. Später wurde eine erneute Nierenfunktionsszintigraphie durchgeführt. Dabei zeigte sich ein Funktionsanteil der linken Niere von nur noch 12\%. Die Flussgeschwindigkeit der linken Nierenarterie betrug 2,4 m/s knapp postostial, dabei kam eine knotige paraaortal links in gleicher Höhe befindliche Fremdgewebsformation zur Darstellung. Eine geplante radiologische Intervention wurde aufgrund des sonographisch geäußerten Verdachts eines Phäochromozytoms storniert.

Die in der Sonographie vaskularisierte Raumforderung paraaortal links bestätigte sich in der Computertomographie (- Abb. 1) als inhomogen Kontrastmittel aufnehmende Raumforderung in direkter Lagebeziehung zur linken Nierenarterie. Die Tumorszintigraphie mit dem Noradrenalinanalogon I-123-mIBG (Metaiodbenzylguanidin) bestätigte einen solitären, mäßig hormonaktiven, mit einem Paragangliom kompatiblen Tumor

(• Abb. 2).

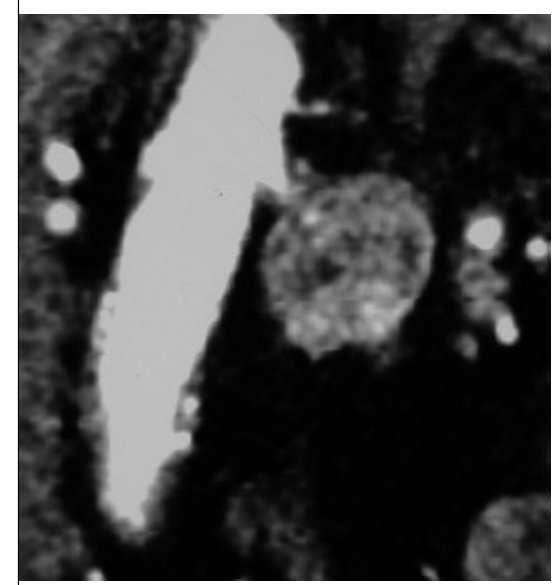

Abb. $1 \Delta$ Bestätigung der links paraaortalen Raumforderung in der Computertomographie

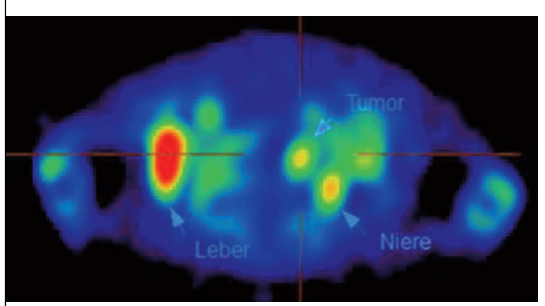

Abb. 2 \ Szintigraphischer Tumornachweis
Symptome eines Phäochromozytoms oft unspezifisch

Der Anteil der Patienten, bei dem ein Phäochromozytom im Rahmen der Hypertonieabklärung diagnostiziert werden kann, liegt bei knapp über 0,1\%. Bei Hinweisen auf eine sekundäre Hypertonieform sollte die diagnostische Abklärung zumindest einmalig klinisch, endokrinologisch und bildgebend vollständig erfolgen, duale Ursachen sekundärer Hypertonie sind zwar selten, aber klinisch bedeutsam. Die Symptome eines Phäochromozytoms sind oft sehr unspezifisch. Kleine retroperitoneale Raumforderungen entziehen sich häufig der sonographischen Diagnostik.

Bei alleiniger (DRG-konformer) Durchführung des Behandlungsauftrags hätte sich im Falle der Patientin angiographisch das Bild einer Nierenarterienstenose geboten, ein Dilatationsversuch bei zugrundeliegendem Phäochromozytom wäre nicht nur ineffektiv gewesen, sondern hätte fatale Konsequenzen nach sich ziehen können.

Quelle: Nephrologe (2010) 5: 230-235 